PREPARED FOR THE U.S. DEPARTMENT OF ENERGY, UNDER CONTRACT DE-AC02-76CH03073

PPPL-3898

PPPL-3898

UC-70

Inverse Bremsstrahlung Stabilization of Noise in the Generation of Ultra-short Intense Pulses by Backward Raman Amplification

by

Richard L. Berger, Daniel S. Clark, Andrei Solodov, Ernest J. Valeo, and Nathaniel J. Fisch

November 2003
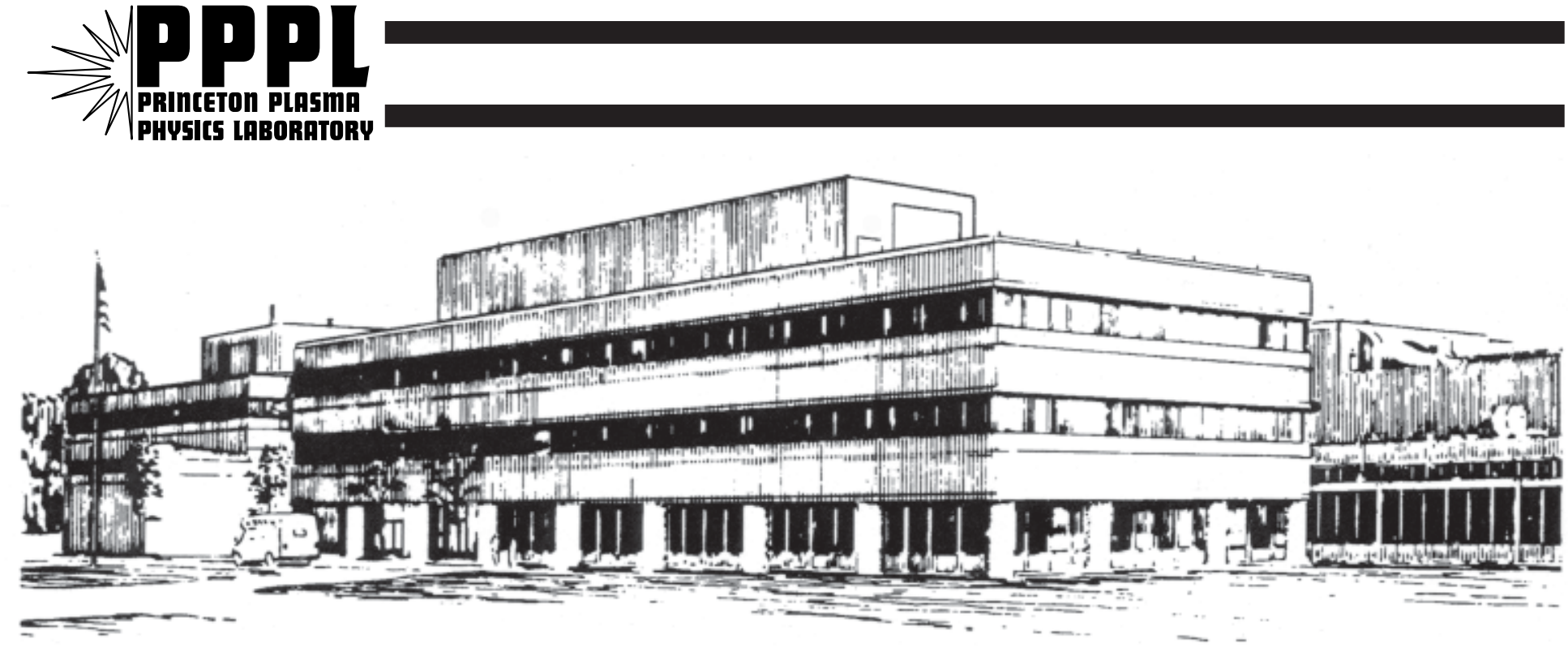

PRINCETON PLASMA PHYSICS LABORATORY PRINCETON UNIVERSITY, PRINCETON, NEW JERSEY 


\section{PPPL Reports Disclaimer}

This report was prepared as an account of work sponsored by an agency of the United States Government. Neither the United States Government nor any agency thereof, nor any of their employees, makes any warranty, express or implied, or assumes any legal liability or responsibility for the accuracy, completeness, or usefulness of any information, apparatus, product, or process disclosed, or represents that its use would not infringe privately owned rights. Reference herein to any specific commercial product, process, or service by trade name, trademark, manufacturer, or otherwise, does not necessarily constitute or imply its endorsement, recommendation, or favoring by the United States Government or any agency thereof. The views and opinions of authors expressed herein do not necessarily state or reflect those of the United States Government or any agency thereof.

\section{Availability}

This report is posted on the U.S. Department of Energy's Princeton Plasma Physics Laboratory Publications and Reports web site in Fiscal Year 2004. The home page for PPPL Reports and Publications is: http://www.pppl.gov/pub_report/

DOE and DOE Contractors can obtain copies of this report from:

U.S. Department of Energy

Office of Scientific and Technical Information

DOE Technical Information Services (DTIS)

P.O. Box 62

Oak Ridge, TN 37831

Telephone: (865) 576-8401

Fax: (865) 576-5728

Email: reports@adonis.osti.gov

This report is available to the general public from:

National Technical Information Service

U.S. Department of Commerce

5285 Port Royal Road

Springfield, VA 22161

Telephone: $1-800-553-6847$ or

(703) $605-6000$

Fax: (703) 321-8547

Internet: http://www.ntis.gov/ordering.htm 


\title{
Inverse bremsstrahlung stabilization of noise in the generation of ultra-short intense pulses by backward Raman amplification
}

\author{
Richard L. Berger, ${ }^{*}$ Daniel S. Clark, ${ }^{\dagger}$ \\ Andrei Solodov, Ernest J. Valeo, and Nathaniel J. Fisch \\ Princeton Plasma Physics Laboratory \\ Princeton University, Princeton, NJ
}

(Dated: October 23, 2003)

\begin{abstract}
Inverse bremsstrahlung absorption of the pump laser beam in a backward Raman amplifier over the round-trip light transit time through the sub-critical density plasma can more than double the electron temperature of the plasma and produce time-varying axial temperature gradients. The resulting increased Landau damping of the plasma wave and detuning of the resonance can act to stabilize the pump against unwanted amplification of Langmuir noise without disrupting nonlinear amplification of the femtosecond seed pulse. Because the heating rate increases with the charge state Z, only low-Z plasmas (hydrogen, helium, or helium-hydrogen mixtures) will maintain a low enough temperature for efficient operation.
\end{abstract}

PACS numbers: $52.40 . \mathrm{Nk}, 52.35 . \mathrm{Mw}$

*Electronic address: berger5@llnl.gov; [permanent address] Lawrence Livermore National Laboratory, Livermore, CA 94551.

†[present address] Lawrence Livermore National Laboratory, Livermore, CA 94551 


\section{INTRODUCTION}

The stimulated backward Raman amplification (BRA) of short seed pulses by long pulse laser beams in centimeter-long, sub-critical plasma has been predicted to produce nearly relativistically intense $\left(I_{\text {seed }} \sim 10^{17} \mathrm{~W} / \mathrm{cm}^{2}\right.$ for $1 \mu \mathrm{m}$ laser light $), 40-100$ femtosecond pulses. The optimum scheme uses transient Raman amplification [1], in which the plasma wave damping rate $\nu_{l}$ is smaller than the Raman growth rate $\gamma_{0}$, the plasma wave frequency $\omega_{p}=\left(\omega_{p e}^{2}+3 k_{p}^{2} v_{e}^{2}\right)^{1 / 2}$, and much smaller than the laser frequency $\omega_{0}$. Here, $\omega_{p e}^{2}=4 \pi e^{2} n_{e} / m_{e}$ is the square of the plasma frequency, $n_{e}$ is the electron density, $m_{e}$ is the electron mass, $k$ is the wavenumber of the plasma wave, and $v_{e}=\left(T_{e} / m_{e}\right)^{1 / 2}$ is the electron thermal velocity where $T_{e}$ is the electron temperature. If $\omega_{p e} \ll \omega_{0}$, then only a small fraction of the pump power is taken up by the Langmuir wave.

The Raman amplification scheme works as follows. Consider a plasma of uniform density and temperature and of length $L$ into which a long-pulse, moderate-intensity laser beam (the pump) enters at $z=0$ and $t=0$. A counter-propagating short pulse light wave (the seed) enters at $z=L$ after a time delay of $L / c$ where $c$ is the speed of light. If the Langmuir wave damping is much less than the Raman growth rate, the seed initially grows at the convective growth rate at the location $L+\left(v_{g l}+v_{g r}\right) t / 2 \sim L-c t / 2$ where $v_{g l(r)}$ is the plasma wave (Raman light seed) group velocity and $c$ is the vacuum velocity of light. As a result, during this linear amplification stage, an effective broadening of the pulse occurs. However, once the pulse has grown sufficiently intense to deplete fully the pump within less than the pulse width, only the front of the pulse is amplified and an effective compression of the pulse occurs. In this pump depletion stage, the pulse power increases as $t^{2}$ and the pulse width decreases as $t^{-1}$. Due to the nonlinear narrowing of the pulse during this stage of amplification, the pulse-pump interaction is robust to large laser bandwidth, plasma inhomogeneity, and plasma wave damping. The optimum density for operation of a backward Raman amplifier is bounded between a low density, $n_{\mathrm{wb}}$, where strong linear Landau damping and wave-breaking occur and a higher density where relativistic ponderomotive Raman forward scatter [2] or modulational instability [3] of the intense seed occur.

Previous work on backward Raman amplification in this regime did not take account of the heating of the plasma by inverse bremsstrahlung absorption of the pump laser beam. If the temperature is constant, BRA works well when the Langmuir wave damping is at a 
minimum. At low $T_{e}$, the plasma wave is collisionally damped at the rate, $\nu_{e i} \sim Z n_{e} T_{e}^{-3 / 2}$ where $\nu_{e i}$ is the electron-ion collision frequency and $Z$ is the charge state of the ions. At high $T_{e}$, strong Landau damping occurs once $k \lambda_{d e}=k v_{e} / \omega_{p e}>0.3$. Collisional damping of the pump by inverse bremsstrahlung occurs at the rate $\nu_{0}=\frac{1}{2}\left(n_{e} / n_{c}\right) \nu_{e i}$ where $n_{c}$ is the critical density at which $\omega_{p e}\left(n_{c}\right)=\omega_{0}$. Minimizing the plasma wave damping determines a plasma temperature of order $100 \mathrm{eV}$ when $n_{e} / n_{c} \sim 0.01$.

At such plasma densities and temperatures, however, the effect of inverse bremsstrahlung heating of the plasma on the amplification process is considerable. For a $1 \mu \mathrm{m}$ laser, a pump intensity of $I=10^{14} \mathrm{~W} / \mathrm{cm}^{2}$ rapidly heats the plasma on the time scale for BRA $\tau_{\text {int }}=2 L / c \sim 50$ ps where $L \sim 1 \mathrm{~cm}$ is the length of the amplifying plasma. For example, if electron heat transport is negligible, a simple calculation shows that $\Delta T_{e} \sim 70 \mathrm{eV}$ after $45 \mathrm{ps}$ in a hydrogen plasma at $0.007 n_{c}$ for which $\nu_{e i}=3.5 \times 10^{11} \mathrm{sec}^{-1}$ initially. This heating reduces the pump and seed absorption over time. More significantly, increasing the electron temperature also increases the Landau damping of the plasma wave significantly as the ratio of the plasma-wave phase velocity to the thermal velocity decreases. This Landau damping increase proves to be an important effect in reducing greatly the amplification of plasma wave noise and in preventing spontaneous SRS from depleting the pump prematurely [5]. Additionally, the spatially nonuniform and temporal nature of this heating introduces an effective Raman detuning gradient due to the temperature dependence of the Langmuir wave dispersion relation. Analogous to detuning using a density gradient or pump chirp [4], this effective detuning gradient can act to stabilize unwanted amplification of Langmuir wave or bremsstrahlung noise with its resulting premature depletion of the pump without disrupting useful nonlinear amplification of the pulse. This paper addresses quantitatively the effects of a spatially and temporally varying plasma temperature on Raman amplification due to inverse bremsstrahlung heating by the pump, in particular, enhancement of the Landau damping rate with heating and detuning of the Raman resonance by thermal gradients.

These simple estimates assume the electron temperature increase is not limited by transport and that the heated distribution maintains a Maxwell-Boltzmann shape. The first assumption is appropriate because the intended scale of operation ( up to several centimeter transverse scale) is much larger than the electron-ion scattering mean free path, $\lambda_{e i} \sim 10^{-3} \mathrm{~cm}$. However, in current experiments [6], transport must be considered because the laser spot size is not much larger than the mean free path. Given that $Z \sim 1$, the elec- 
tron self-collisions which operate to maintain the Maxwellian shape have a collision time, $\tau_{e e} \sim \tau_{e i} \sim 3$ ps. Thus the Maxwellian assumption made in this work is reasonable. In subsequent work, this assumption will be tested with the expectation that any calculated reduction in the Landau damping rate will allow more flexibility in choosing parameters.

This paper is organized as follows. Sec. II introduces the model used to describe the Raman amplification process and simultaneous plasma heating. Sec. III addresses the effect of increased Landau damping on the growth of backscatter from noise concomitant with plasma heating. Sec. IV extends these thermal effects by considering the effect of temperature variations in detuning the Raman resonance. Sec. V presents the results of simulations with pF3d. Sec. VI considers the possible effects of nonlinearities in Landau damping on amplification, and Sec. VII summarizes and concludes.

\section{MODEL}

The Raman interaction is simulated using the pF3d code [7] designed for laser plasma interactions in sub-critical plasma. Since this work is concerned with effects that are essentially one dimensional, the pF3d reduced system consists of three enveloped equations for the pump $A_{0}$, the Raman scattered light $A_{r}$, and the plasma wave $n_{p}$. Although hydrodynamic motion is allowed and calculated, no significant density or flow velocity develops over the 50 ps interaction time. However, the electrons are heated by the absorption of the pump energy. The equations of this pF3d model are

$$
\begin{aligned}
\left(\frac{\partial}{\partial t}+v_{g 0} \frac{\partial}{\partial z}+\nu_{0}\right) A_{0} & =\frac{-i}{4} \frac{\omega_{p e}^{2} n_{p} A_{r}}{\omega_{0} n_{e}} \\
\left(\frac{\partial}{\partial t}+v_{g r} \frac{\partial}{\partial z}+\nu_{r}\right) A_{r} & =\frac{-i}{4} \frac{\omega_{p e}^{2} n_{p}^{*} A_{0}}{\omega_{r} n_{e}} \\
\left(\frac{\partial}{\partial t}+v_{g l} \frac{\partial}{\partial z}+\nu_{l}+i \delta \omega_{p}\right) n_{p} & =\frac{-i k_{p}^{2} n_{e}}{4 \omega_{p}} \frac{e A_{0}}{m_{e} c} \frac{e A_{r}^{*}}{m_{e} c}+S_{p}(z, t) \\
\frac{3}{2}\left(\frac{\partial}{\partial t}+U_{e} \nabla\right) T_{e}+T_{e} \nabla U_{e} & =\frac{m_{e}}{2} \nu_{e i} v_{0}^{2}-n_{e}^{-1} \nabla q_{e}
\end{aligned}
$$

Here $\nu_{0}, \nu_{r}$, and $\nu_{l}$ are the respective damping rates of the pump, Raman light, and Langmuir wave; $\nu_{e i}$ is again the electron-ion collision frequency. In Eq. $4, U_{e} \ll v_{e}$ is the electron flow velocity, and $q_{e} \sim-n_{e} v_{e} \lambda_{e i} \nabla T_{e} \gg n_{e} T_{e} U_{e}$ is the electron heat flow. If the 
pump laser spot size is $\sim 1 \mathrm{~cm}$, then the lateral heat flow is unimportant; that is, because $n_{e} T_{e} /\left(\nabla q_{e}\right) \sim L_{\perp}^{2} /\left(v_{e} \lambda_{e i}\right) \sim 3 \times 10^{-6}$ sec, the time scale for loss of energy from the directly heated plasma is orders of magnitude longer than twice the transit time of the laser pulse axially across the plasma, $\sim 50$ ps. The axial heat flow is also unimportant because, as will be shown subsequently, the axial gradients induced by the inverse bremsstrahlung heating are also $\sim 1 \mathrm{~cm}$.

For electron temperatures of the order of $100 \mathrm{eV}$, the jitter velocity $v_{0}=e E / m_{e} \omega_{0}$ of an electron in the pump laser electric field $E$ is of the same order as or greater than the electron thermal velocity $v_{e}=\sqrt{T_{e} / m_{e}}$. For example, $v_{0}=v_{e}=2.7 \times 10^{8} \mathrm{~cm} / \mathrm{s}$ for $1.054 \mu \mathrm{m}$ laser light at $10^{14} \mathrm{~W} / \mathrm{cm}^{2}$ and $T_{e}=40 \mathrm{eV}$. Under these conditions, the collision frequency is reduced [9] with the dominant "strong field" effect obtained by setting $v_{e}^{2} \rightarrow v_{e}^{2}+v_{0}^{2}[16]$. The frequencies and wave numbers of the three waves, $\omega_{0, r, p}$ and $k_{0, r, p}$, are chosen to have exact resonance given the plasma density and temperature in a homogeneous plasma. That is the phase matching conditions

$$
\begin{aligned}
& \omega_{p}=\omega_{0}-\omega_{r} \\
& k_{p}=k_{0}-k_{r}
\end{aligned}
$$

are satisfied. For densities and temperatures varying slowly in space and time, exact resonance is chosen in this study at the mean density and initially uniform temperature. The phase mismatch that develops in space and time is accounted for by the frequency detuning term, $\delta \omega_{p}$, in Eq. (3). The Landau damping (with the kinetically correct dispersion), the collisional damping, the inverse bremsstrahlung absorption, and the heating rate are recalculated after every hydrodynamic time step. The source $S_{p}(z, t)$ of plasma waves in Eq. (4) has a magnitude chosen such that uncoupled plasma waves will be driven to the thermal fluctuation level. It is uncorrelated along $z$. The correlation time for the source is less than the plasma wave damping time.

The linearized solution of Eqs. (2)-(3) in spatially homogeneous plasma has exponentially growing solutions above thresholds set by the damping rates, $\nu_{r}$ and $\nu_{l}$. If these damping rates are negligible, the growth rate is $\gamma=\gamma_{0}$ where

$$
\gamma_{0}=\frac{1}{2} \sqrt{\omega_{0} \omega_{p}} \frac{v_{0}}{c}
$$

and the approximations are made that $\omega_{p}=\omega_{p e}, k_{p}=2 k_{0}=2 \omega_{0} / c$, and $\omega_{r} \sim \omega_{0}$. Otherwise, the convective instability threshold obtained from Eqs. 2 and 3 is $\gamma_{0}^{t h}=\sqrt{\nu_{r} \nu_{l}}$. If the plasma 
wave is collisionally damped and $\gamma_{0} \gg \nu_{l} \sim n_{c} / n_{e} \nu_{r}>\gamma_{0}^{t h}$, this instability grows at the rate

$\gamma_{0}$ from an initial source at the location $z_{\text {init }}+\left(v_{\text {gr }}+v_{\text {gl }}\right)\left(t-t_{\text {init }}\right) / 2 \sim z_{\text {init }}-c\left(t-t_{\text {init }}\right) / 2$ where the latter applies because $n_{e} \ll n_{c}$ and $v_{g l} \ll\left|v_{g r}\right| \sim c$. In a plasma near thermal equilibrium, the fluctuation amplitudes of the light and plasma waves fields (so called noise) are so small that about ten $e$-foldings of amplitude are required to deplete the pump energy [8]. Because the plasma is optically thin, the bremsstrahlung source for $A_{r}$ is smaller than the source arising from Thomson scatter off Langmuir fluctuations[8] and is not included in our simulations. The initial amplitude of the sub-picosecond seed pulse is much larger and needs only a couple of e-foldings to deplete the pump. For effective Raman amplification, the amplification of noise must then be controlled using density gradients or chirping of the pump frequency while retaining enough gain for the seed to deplete the pump and compress nonlinearly.

\section{EFFECT OF ENHANCED LANDAU DAMPING WITH HEATING}

Consider again a uniform plasma slab of length $L$ where a seed, launched at $t=L / c$, reaches the pump depletion stage within a short time. Then the seed will propagate at velocity $-c$ while the peak of the noise amplified from a source at $z_{\text {init }}$ propagates at velocity $-c / 2$. The seed pulse will overtake the peak amplitude of any small amplitude noise pulse that originates at a distance larger than $2 L / 3$ from the boundary at $z=0$. Thus, the maximum amplitude gain for noise in the plasma of length $L$ is $\exp \left(G_{0}\right)$ with $G_{0}=4 \gamma_{0} L / 3 c$. For parameters of interest, $\gamma_{0} \sim 2.3 \times 10^{12} \mathrm{sec}^{-1}$ and $L \sim 0.7 \mathrm{~cm}$, so that $G_{0} \sim 72$ and strong depletion of the pump by amplified noise will occur.

For plasma densities of interest $\left(n_{e} \leq .01 n_{c}\right)$, Landau damping of the plasma wave increases rapidly as the electron temperature increases above $50 \mathrm{eV}$ as shown in Fig. 1. Here, the Langmuir wave damping rate, including both collisional (with $Z=1$ ) and Landau damping, is shown as a function of the electron temperature. Also shown is the collisional damping which decreases as $T_{e}^{-3 / 2}$. Once $T_{e} \sim 80 \mathrm{eV}$, the linear Landau damping becomes comparable to the SRS convective growth rate $\gamma_{0}$ for $I=10^{14} \mathrm{~W} / \mathrm{cm}^{2}$ and $n_{e}=0.007 n_{c}$. At the higher temperatures where $\gamma_{0}<\nu_{l}$, the convective growth rate is reduced from $\gamma_{0}$ to $\gamma_{0}^{2} / \nu_{l}$, and the peak amplitude of backscattered wave propagates at nearly $v_{g r} \sim-c$ with a gain of $\exp \left(G_{s d}\right)$ for $G_{s d}=\gamma_{0}^{2} L / \nu_{l}\left|v_{g r}\right| \sim 54 \gamma_{0} / \nu_{l}$ for $L=0.7 \mathrm{~cm}$. Thus, in the absence of 


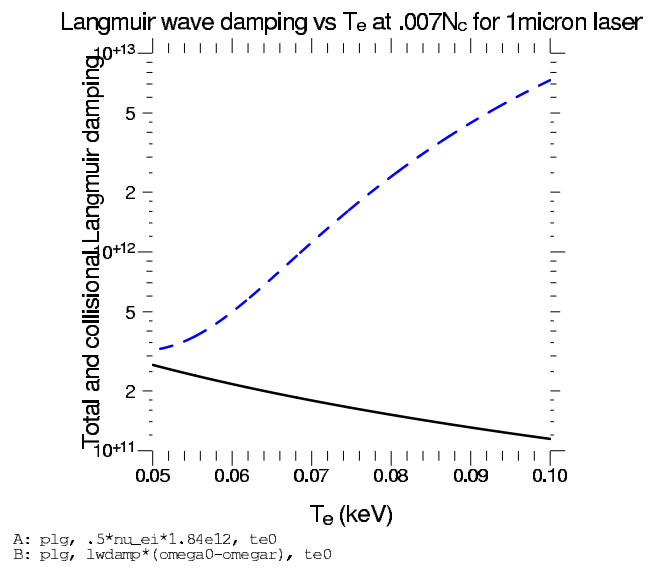

FIG. 1: Langmuir wave damping rate plotted as a function of electron temperature. The total damping rate it given by the dashed curve and the collisional contribution by the solid curve. The plasma electron density, $n_{e}=.007 n_{c}$ and the charge state $Z=1$.

chirping or density gradients, only a plasma wave damping $\nu_{l}$ an order of magnitude larger than $\gamma_{0}$ will reduce amplification of the noise to acceptable values.

Note that, at the time the seed is launched, the plasma near $z=L$ (where the seed enters) has not been heated. Thus, the Langmuir wave damping rate is at its minimum and the seed initially convectively amplifies at the fastest rate possible, $\gamma_{0}$, and will reach the pump depletion level before the temperature increase slows that rate if the seed amplitude is large enough. This requirement on the seed amplitude is that it be able to reach the pump depletion level before the Landau damping rate exceeds the pulse bandwidth, $\Delta \omega=2 \pi / \tau_{\text {seed }}$. The amplification of Langmuir noise in the vicinity of $z=0$, however, will experience the strongest temperature effects and hence be most strongly suppressed by the increased Landau damping rate. This effect could potentially be very beneficial for Raman amplifiers. 


\section{RAMAN DETUNING DUE TO TEMPERATURE VARIATION}

In addition to the enhancement of the Landau damping rate with heating, the axial temperature gradient and accompanying detuning of the Raman resonance introduced by plasma heating is an important effect. The linear theory of SRS in a temperature and density gradient, applicable to the noise amplification at least during the early stages, is well developed [10]. For strong enough time-stationary gradients, the amplitude gain is $\exp \left(G_{\nabla}\right)$ where

$$
G_{\nabla}=\pi \gamma_{0}^{2} / \kappa^{\prime}\left|v_{g l} v_{g r}\right|
$$

Here,

$$
\kappa^{\prime}=\left.\frac{d}{d z}\left(k_{0}-k_{r}-k_{p}\right)\right|_{\kappa=0},
$$

is the rate of detuning from Raman resonance at the point of exact resonance $\left(\kappa=k_{0}-k_{r}-\right.$ $\left.k_{p}=0\right)$. The plasma wave is most sensitive to the gradients in temperature and density. From the fluid dispersion relation for Langmuir waves,

$$
\kappa^{\prime}=-\frac{k_{p}}{2}\left(\frac{d}{d z} \ln T_{e}+\frac{1}{3 k_{p}^{2} \lambda_{d e}^{2}} \frac{d}{d z} \ln n_{e}\right) .
$$

From this equation, one notes that, for long wavelengths, a density gradient is more effective than a temperature gradient in suppressing Raman amplification. The neglect of the damping of the plasma wave requires that $G_{\nabla}<G_{s d}$ from which one obtains the condition, $\nu_{l} / \omega_{p}<3 / 2 k_{p}^{2} \lambda_{d e}^{2} L\left(d \ln T_{e} / d z\right)$.

In the case of Raman amplification in which the plasma temperature is time dependent as well as spatially dependent, the more general theory applicable to parametric growth in media slowly varying in space and time must be considered [11]. In place of Eq. 6, the gain exponent becomes

$$
G_{z t}=\pi \gamma_{0}^{2} /|B|
$$

where

$$
B=\left(\partial_{t}^{2}+\left(v_{g l}+v_{g r}\right) \partial_{t} \partial_{z}+v_{g r} v_{g l} \partial_{z}^{2}\right) \phi
$$

and $\phi=\phi_{0}-\phi_{r}-\phi_{l}$ is the phase mismatch between the three waves such that the usual relations hold, namely, the wavenumber $k_{j}=\partial_{z} \phi_{j}$ and the frequency $\omega_{j}=-\partial_{t} \phi_{j}$ for $j=$ $0, r, l$. In the present case, the electron density is constant in space and time, the temperature varies temporally and spatially because of inverse bremsstrahlung heating, and the pump 
frequency is chirped. The phase of the pump is taken to be $\omega_{0} t+q\left(\gamma_{0} t\right)^{2} / 4$ and the linear gain becomes $\exp \left(G_{z t}\right)$ where

$$
G_{z t}=\frac{\pi \gamma_{0}^{2}}{\left(q \gamma_{0}^{2}+3 k_{p}^{2} \lambda_{d e}^{2} \omega_{p} \frac{\partial}{\partial t} \ln T_{e}\right)} .
$$

The inclusion of the time variation as well as the spatial variation reduces the gain exponent (if $q=0$ ) by an important factor of two. If $\dot{T}_{e}=T_{e}^{\prime}=0$, Eq. (11), reduces to the result obtained previously for pure frequency detuning [4], namely $G_{z t}=\pi / q$. Also, this equation shows that one might choose the sign of a pump frequency chirp and/or a density gradient to enhance or reduce the effect of the temperature gradient. An estimate of the temperature gradients appearing in these equations can be found from Eq. (4). Including this strong field correction but neglecting the heat flow and for a given uniform plasma density and charge state, the solution to Eq. (4) is

$$
T_{e}(t)=-T_{o s c}+T_{e}\left(t_{0}\right)\left[\left(1+\frac{T_{o s c}}{T_{e}\left(t_{0}\right)}\right)^{\frac{5}{2}}+\frac{5}{6} \frac{T_{o s c}}{T_{e}\left(t_{0}\right)} \nu_{e i}^{0}\left(t-t_{0}\right)\right]^{\frac{2}{5}} .
$$

The laser intensity is assumed constant, a good assumption given the weak inverse bremsstrahlung absorption. Here $\nu_{e i}^{0}=4 \sqrt{2 \pi / m_{e}} e^{4} Z n_{e} \ln (\Lambda) / 3 T_{e}\left(t_{0}\right)^{3 / 2}$ is the "weak-field" electron-ion collision frequency at the initial time $t_{0} . T_{e}\left(t_{0}\right)$ is the initial, uniform electron temperature, and $T_{o s c}=m_{e} v_{0}^{2}$. In Fig. 2, this solution is plotted for $T_{e}\left(t_{0}\right)=50 \mathrm{eV}$, $n_{e}=0.007 n_{c}$, and $Z=1$. Because heating begins at a given location $z$ only after the pump has propagated to that point, the space-time dependence of the temperature (neglecting conduction) is simply given by $T_{e}(z, t)=T_{e}\left(t-z / v_{g 0}\right)$. Due to the temperature dependence of the Langmuir wave dispersion relation, the axial temperature gradient introduced by the spatio-temporal dependence of $T_{e}$ can act as an effective detuning of the Raman resonance. From Eq. (12), the temperature gradient is

$$
\frac{d}{d t} \ln T_{e}=\frac{1}{3}\left(\frac{T_{e}(0)}{T_{e}(t)}\right)^{5 / 2}\left(1+\frac{T_{o s c}}{T_{e}(t)}\right)^{-3 / 2} \frac{T_{o s c}}{T_{e}(0)} \nu_{e i}^{0} .
$$

For the same parameters as above, $d \ln T_{e} / d z \sim 1 \mathrm{~cm}^{-1}$. This estimate agrees well with the results of a pF3d simulation in a hydrogen plasma shown in Fig. 3. Assuming the density gradient is zero, as it is in our simulations, we use Eq. (11) to obtain, $G_{z t} \sim 10$ for $T_{e} \sim 90 \mathrm{eV}$ for which $k_{p} \lambda_{d e} \sim 0.3$. For these parameters, the damping is weak enough (by a factor of five) for the gradient gain exponent (Eq. 11) to be the smallest exponent. 


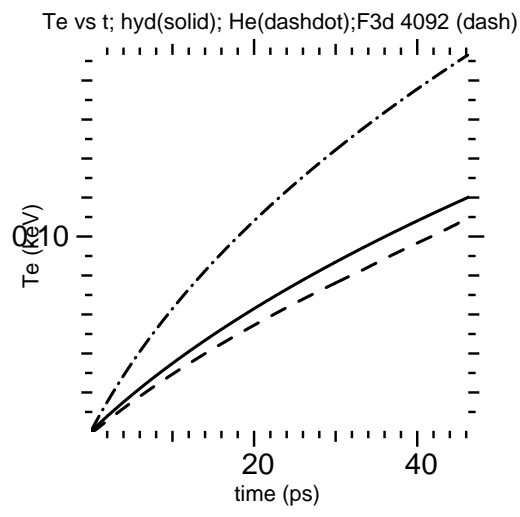

FIG. 2: The electron temperature as a function of time. The solid and dash-dot curves are the solution given in Eq. (5) for hydrogen and helium respectively with $T_{e}(0)=.05 \mathrm{eV}, n_{e}=.007 n_{c}$, $I=1 \times 10^{14} \mathrm{~W} / \mathrm{cm}^{2}$, and laser wavelength, $\lambda_{0}=1053 \mathrm{~nm}$. The dashed curve is the pF3d solution with the strong field reduction.

Thus, in this example, gradient stabilization is the dominant limiting factor. Because the damping rate increases rapidly with $k_{p} \lambda_{d e}$, Landau damping will determine the gain as the plasma heats further. To achieve stronger damping and weaker gain in the front half of the plasma where it is most effective in reducing unwanted noise amplification a gradient in charge state might be desirable, which could be achieved by using mixtures of helium and hydrogen. Although the temperature gradient reduces the gain to nearly an acceptable value, it is still not sufficient to suppress noise amplification entirely in ionized hydrogen plasma. In the simulations below, some frequency chirp is still required to suppress the noise amplification, although much less than needed without the heating induced gradient. 


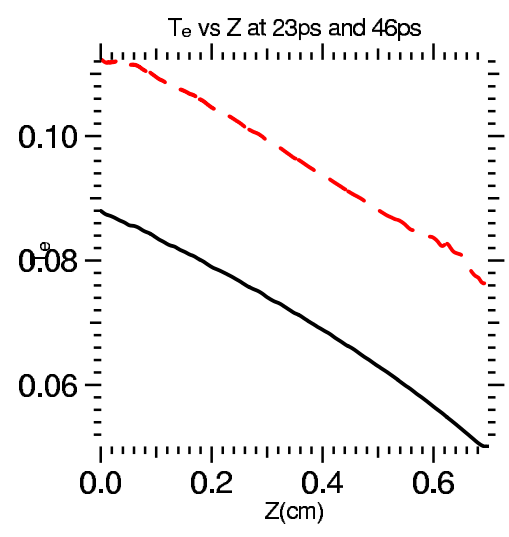

FIG. 3: The electron temperature $(\mathrm{keV})$ is shown along the propagation direction at 23 ps when the seed is launched and at $46 \mathrm{ps}$ when the amplified seed nears the boundary of the plasma at $\mathrm{z}=0$. The simulation parameters are $T_{e}(0)=.05 \mathrm{eV}, n_{e}=.007 n_{c}, I=1 \times 10^{14} \mathrm{~W} / \mathrm{cm}^{2}$, and pump laser wavelength, $\lambda_{0}=1053 \mathrm{~nm}$.

\section{SIMULATION RESULTS}

Without the gradients induced by the heating, spontaneous SRS will grow from noise to nonlinear levels and partially deplete the pump as well as introduce enhanced Langmuir wave noise and a non-Maxwellian tail of hot electrons. Such effects reduce the seed gain and seed pulse compression. One solution is to introduce a chirp in the pump frequency. If the chirp is small, the noise will prematurely deplete the pump as shown in Figs. 4 and 5 . In Fig. 4, the pump has been severely depleted by the spontaneous backscatter at the time the pump has just traversed the plasma and the seed is launched into the plasma. In Fig. 5, the pump and seed are shown at the time when the seed has traversed half the plasma. The seed intensity is about one tenth the value it attains when no spontaneous backscatter is introduced. 


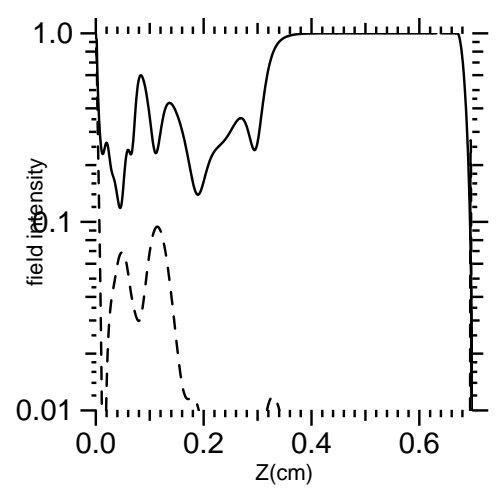

FIG. 4: The amplitude of $A_{0}$ (solid) and the reflected light, $A_{r}$ (dashed) at $23 \mathrm{ps}$ with q=0.08 for an unheated hydrogen plasma. Other parameters are $T_{e}=.05 \mathrm{eV}, n_{e}=.007 n_{c}, I=1 \times 10^{14} \mathrm{~W} / \mathrm{cm}^{2}$, $\lambda_{0}=1053 \mathrm{~nm}, I_{\text {seed }}=2.5 \times 10^{13} \mathrm{~W} / \mathrm{cm}^{2}$, and the initial seed pulse width, $\tau=40 \mathrm{fs}$.

Heating alone without pump chirp may be adequate to suppress the noise amplification in the simulations as is illustrated in Fig. 6 and 7 for a helium plasma for which the inverse bremsstrahlung heating rate is twice that of hydrogen at the same temperature. In Fig. 6, the laser intensity is unaffected by the very weak growth of spontaneous backscatter at the time that the seed is just encountering the pump at $z=L$. In Fig. 7, the seed has amplified four hundredfold and depleted the pump. For a hydrogen plasma, some pump chirp in addition to the heating is required to achieve similar gain.

As discussed earlier in the context of temperature gradients, it is well known [10] that a linear gradient in the wavenumber matching, that is $k_{0}-k_{r}-k_{p}=\kappa^{\prime} z$, leads to a convective saturation of SRS amplitude at the value $\exp \left(\pi \gamma_{0}^{2} /\left|\kappa^{\prime} v_{g r} v_{g l}\right|\right)$ even when the instability is above the absolute instability damping threshold defined by $\gamma_{0} / \sqrt{\left(\left|v_{g r} v_{g l}\right|\right)=}$ $1 / 2\left(\nu_{l} /\left|v_{g l}\right|+\nu_{r} /\left|v_{g r}\right|\right)$. However, random or periodic density inhomogeneity can restore the absolute instability [12] which again prematurely depletes the pump from amplified noise. Another benefit of heating is to increase the absolute instability damping threshold. Then 


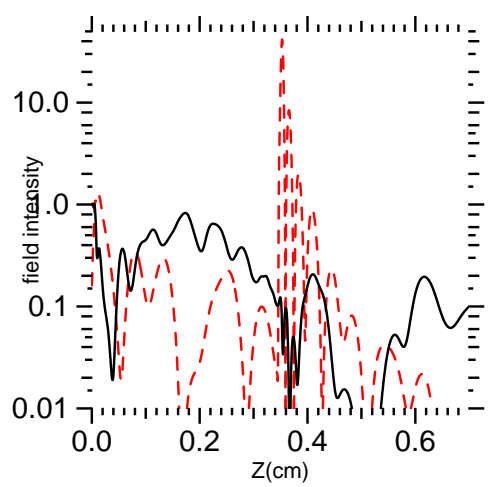

FIG. 5: The amplitude of $A_{0}$ (solid black) and the reflected light, $A_{r}$ (dashed red) of $A_{0}$ at $35 \mathrm{ps}$ with $\mathrm{q}=0.08$ for an unheated hydrogen plasma. Other parameters are $T_{e}=.05 \mathrm{eV}, n_{e}=.007 n_{c}$, $I=1 \times 10^{14} \mathrm{~W} / \mathrm{cm}^{2}, \lambda_{0}=1053 \mathrm{~nm}, I_{\text {seed }}=2.5 \times 10^{13} \mathrm{~W} / \mathrm{cm}^{2}$, and the initial seed pulse width, $\tau=40 \mathrm{fs}$.

the presence of density inhomogeneity cannot restore absolute growth.

A summary of these results is shown in Fig. 8. For the parameters of these simulations, $\left(I_{\mathrm{L}}=1 \times 10^{14} \mathrm{~W} / \mathrm{cm}^{2}, T_{e}(0)=.05 \mathrm{keV}, Z=1, n_{e} / n_{c}=.007, L=0.7 \mathrm{~cm}\right)$, the best possible seed intensity gain of 1800 , corresponding to a seed intensity of $5 \times 10^{16} \mathrm{~W} / \mathrm{cm}^{2}$, is found by turning off the heating, the pump frequency chirp, and the thermal noise source for the Langmuir waves. The initial seed intensity was 0.25 of the initial pump intensity. Without heating but with Langmuir wave noise, the seed gain decreases an order of magnitude but increases in response to increasing pump frequency chirp ( $\mathbf{a}$ ) as was shown previously [4]. Chirp values corresponding to $q \sim 0.2$ are required to achieve good seed gains of $\sim$ 1600. With inverse bremsstrahlung heating, the combined effect of increased Langmuir wave damping and the temperature gradient detuning are nearly sufficient to suppress the spontaneous SRS and only a small amount of pump chirp restores nearly full gain as shown by the blue $\bullet$. The magnitude of the chirp needed depends logarithmically on the magnitude 


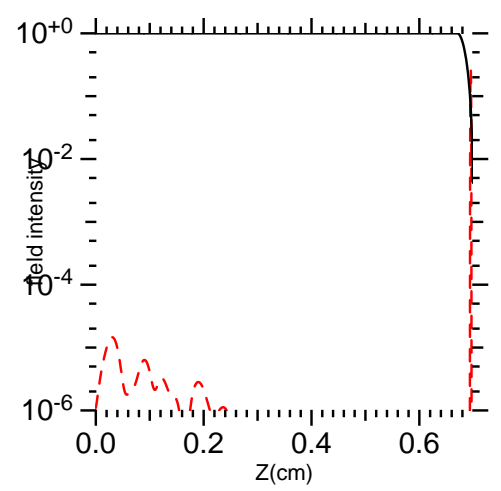

FIG. 6: The amplitude of the pump $A_{0}$ (solid black) and the backscattered light $A_{r}$ (dashed red) at $23 \mathrm{ps}$ for a heated helium plasma with no pump chirp. Other parameters are $T_{e}(0)=.05 \mathrm{eV}$, $n_{e}=.007 n_{c}, I=1 \times 10^{14} \mathrm{~W} / \mathrm{cm}^{2}, \lambda_{0}=1053 \mathrm{~nm}, I_{\text {seed }}=2.5 \times 10^{13} \mathrm{~W} / \mathrm{cm}^{2}$, and the initial seed pulse width, $\tau=40 \mathrm{fs}$.

of the Langmuir noise. Increasing the amplitude of the Langmuir wave noise one hundredfold requires that the chirp $q$ be increased to 0.3 to achieve similar seed gain if no heating occurs.

The suppression of spontaneous SRS by heating is not robust to the introduction of random, stationary density perturbations and more pump chirp is required to restore adequate gain as is shown by the cyan $\downarrow$ curve. For this curve, the rms density amplitude was $3 \%$ of the background and the correlation length was $\sim 0.03 \mathrm{~cm}$. However, in all cases less chirp is required when the heating is accounted for.

Eq. (11) does not account for Langmuir wave damping under the assumption that increased damping will broaden the resonance as it lowers the growth rate such that the overall gain will remain the same as without damping. Such a result applies in stationary plasmas when the wavenumber mismatch varies linearly near resonance. In the case presented here, this assumption is not bourn out by the pF3d simulations. If the plasma wave damping is set to zero in the $\mathrm{pF} 3 \mathrm{~d}$ simulations while retaining the temperature gradient 


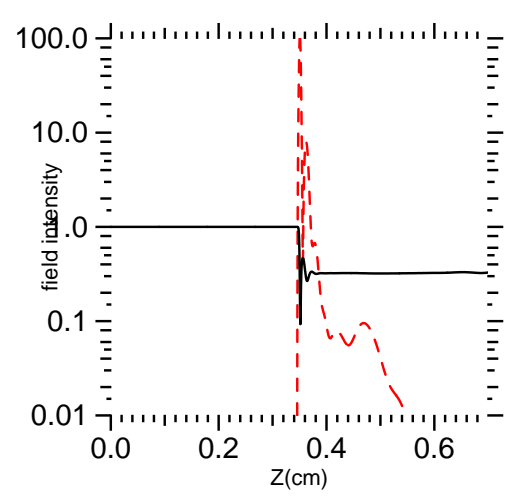

FIG. 7: The amplitude of the pump $A_{0}$ (solid black) and the backscattered light $A_{r}$ (dashed black) at 35 ps.for a heated helium plasma with no pump chirp. Other parameters are $T_{e}(0)=.05 \mathrm{eV}$, $n_{e}=.007 n_{c}, I=1 \times 10^{14} \mathrm{~W} / \mathrm{cm}^{2}, \lambda_{0}=1053 \mathrm{~nm}, I_{\text {seed }}=2.5 \times 10^{13} \mathrm{~W} / \mathrm{cm}^{2}$, and the initial seed pulse width, $\tau=40 \mathrm{fs}$.

effects, the seed gain drops from 1000 (without pump chirp) to 400 because of large spontaneous SRS growing from noise. On the other hand, if the detuning from heating is set to zero but the Landau damping is retained in the pF3d simulations, even more spontaneous SRS grows and the seed gain drops to 120, thus verifying our earlier theoretical estimates that gradient detuning is more important than Landau damping reduction of the growth rate for fully-ionized hydrogen plasmas. The introduction of pump frequency chirp again restores adequate gain for each of these idealized cases (as shown in Fig. 8), with less chirp required for the case with gradient detuning and without damping than the case with damping and without gradient detuning. Simulations with fully-ionized helium plasmas, where the heating is more pronounced, required no pump chirp to achieve a nearly optimum seed gain of 1500 . 


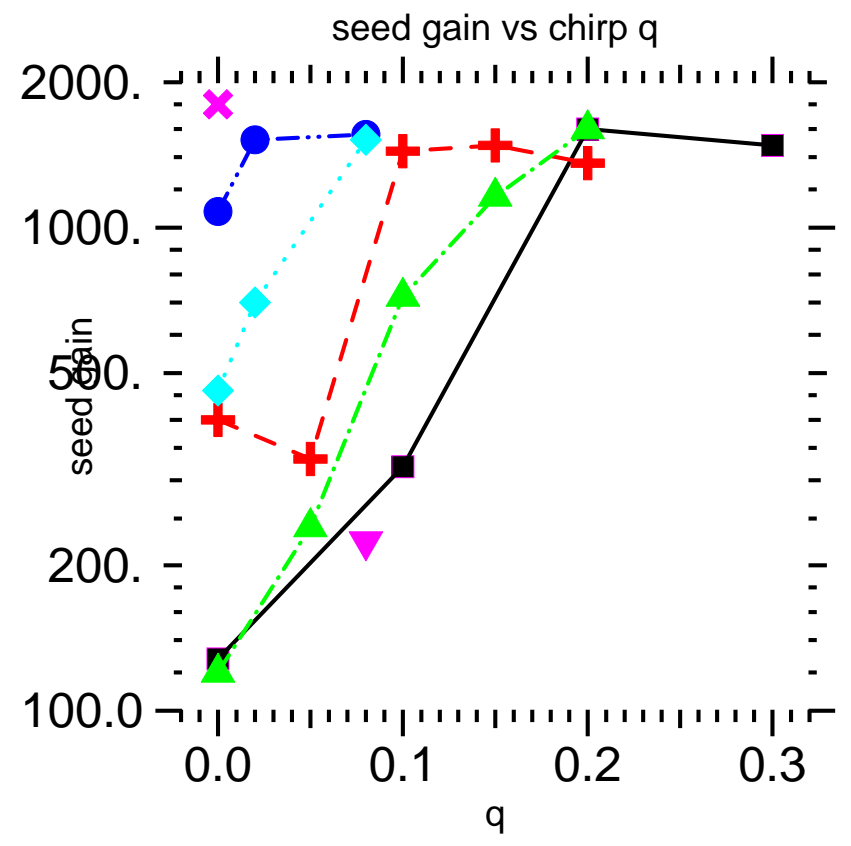

FIG. 8: The gain is shown as a function of the chirp strength characterized by $q$ for no heating and no density perturbations (black $\mathbf{0}$ ), for heating and no density perturbations (blue •), for heating and density fluctuations at a 3 percent rms level (cyan $\downarrow$ ). Also shown are points with no chirp and no Langmuir wave noise $($ purple $\times$ ) and for chirp, density perturbations, and no heating (purple $\boldsymbol{\nabla}$ ). The effect of Landau damping without frequency mismatch (green $\boldsymbol{\Delta}$ ) and frequency mismatch without Landau damping $($ red +$)$ are also shown. Without heating and density perturbations, stronger chirp $(q \sim 0.2-0.25)$ is required to achieve the nearly optimum gain. The simulation parameters are $T_{e}(0)=.05 \mathrm{eV}, n_{e}=.007 n_{c}, I=1 \times 10^{14} \mathrm{~W} / \mathrm{cm}^{2}, \lambda_{0}=1053 \mathrm{~nm}$, $I_{\text {seed }}=2.5 \times 10^{13} \mathrm{~W} / \mathrm{cm}^{2}$. 


\section{EFFECT OF NONLINEARITIES IN LANDAU DAMPING}

Finally, we consider the possible influence of nonlinear effects on the Landau damping of the Langmuir wave. In the pump depletion limit, the Langmuir wave is driven by the pump and seed ponderomotive force to an amplitude, $a_{l} \sim a_{0}$ such that the plasma wave will break if the electron density is lower than $.004 n_{c}$, only a factor of two lower than the density used in our simulations [13]. Here, $a_{0}=e E_{0} / m_{e} c \omega_{0}$ and $a_{p}=e E_{l} / m_{e} c \sqrt{\omega_{0} \omega_{p}}$. The wave-breaking condition corresponds to the plasma wave frequency, $\omega_{p}$, being equal to the bounce frequency, $\omega_{b}=\sqrt{k_{p} e E_{l} / m_{e}}$, of an electron trapped in the Langmuir wave electric field, $E_{l}$. Because the linear Landau damping rate, $\gamma_{l}$, under consideration is at least ten times smaller than $\omega_{p}$, the bounce frequency for the plasma wave driven by the BRA will exceed the linear damping rate. In that case, one might expect the actual damping rate for this interaction to be smaller than used in our simulations as the distribution is flattened near the phase velocity of the plasma wave. On the other hand, this effect also introduces nonlinear frequency shifts[14] that are also not accounted for in our simulations. However, once the seed is amplified to the pump depletion limit, the BRA process is insensitive to the actual value of the damping.[15] More important is the modification of the damping by large amplitude waves driven by spontaneous SRS because these simulations may underestimate the spontaneous SRS growth by overestimating the Landau damping. For the case where the heating is just sufficient to suppress spontaneous SRS, the Langmuir wave amplitude for the spontaneous SRS is about $0.06 a_{0}$ for which $\omega_{b}$ is 0.24 of the value at the pump depletion level. This value will still exceed the linear Landau damping and some modification of the damping should be expected. There are several effects that can reduce the nonlinear modification of the distribution: electron-electron collisions that drive the distribution towards a Maxwellian, electron-ion collisions that scatter the electron out of the resonant region before a bounce oscillation is complete, and the increasing number of electrons at the phase velocity caused by the inverse bremsstrahlung heating. All of these effects rely on the rather small collision frequency, $\nu_{e i} \sim \nu_{e e} \sim 3 \times 10^{11} \mathrm{sec}^{-1}$. The electron-electron scattering is the most effective of these and results in the condition for the validity of linear Landau damping that $\omega_{b} / \omega_{p e}<$ $\left(\nu_{e e} / \omega_{p e}\right)^{1 / 3}\left(k_{p} \lambda_{d e}\right)^{5 / 3} \sim .02$. For the case of helium with good seed gain however, the Langmuir wave amplitude for the spontaneous SRS has a small bounce frequency, $2 \%$ of the pump depletion value and less than the heated linear Landau rate. For this case our fluid 
simulations should be adequate.

\section{CONCLUSIONS}

The inverse bremsstrahlung heating of the electrons by the pump laser beam has been shown to introduce beneficial time-varying electron temperature gradients along the pump propagation direction. The temperature increase with time causes the linear Landau damping of the Langmuir wave to increase with time with the result that the spatial and temporal growth rate for stimulated Raman scatter decreases. Because the seed is launched into plasma that the pump has not had time to heat, the linear stage of the BRA seed amplification is not adversely affected provided the seed intensity is large enough to reach the pump depletion level in a short time. However, the unwanted amplification of thermal plasma wave fluctuations to pump depletion levels may be reduced. Our estimates and simulations indicate that the reduction from increased Landau damping is not sufficient for ionized hydrogen plasma. However, because the temperature increase is proportional to the charge state, $Z$, helium or hydrogen-helium mixtures may provide sufficient increase in the Landau damping. The plasma wave amplitudes reached in the pF3d simulations are sufficiently large, even below wavebreaking, that modifications to the electron distribution function may invalidate the use of linear Landau damping rates. Nonetheless, the reduction of the SRS gain will survive because the principal cause of the reduction is spatial and temporal detuning caused by the temperature dependence of the Langmuir wave dispersion. This gain reduction is calculated and confirmed by pF3d simulations. The theory and simulations also show that pump frequency chirp and heating can be used together for added gain control.

\section{Acknowledgments}

We are pleased to acknowledge helpful input from V. Malkin and E. A. Williams. This work was supported by DARPA and the U. S. Department of Energy contracts DE-AC0276-CHO3073 and W-7405-Eng-48. 
[1] V. M. Malkin, G. Shvets, and N. J. Fisch, Phys. Rev. Lett., 82, 4448 (1999)

[2] T. M. Antonsen and P. Mora, Phys. Rev. Lett., 69, 2204 (1992)

[3] C. E. Max, J. Arons, and A. B. Langdon, Phys. Rev. Lett., 33, 209 (1974)

[4] V. M. Malkin, G. Shvets, and N. J. Fisch, Phys. Rev. Lett., 84, 1208 (2000)

[5] D. S. Clark and N. J. Fisch, Phys. Plasmas 10, 3363 (2003)

[6] Y. Ping I. Geltner S. Suckewer, Phys. Rev. E. 67, 0164XX (2003)

[7] C. H. Still, R. L. Berger, A. B. Langdon, D. E. Hinkel, L. J. Suter, and E. A. Williams, Phys. Plasmas 7, 2023 (2000)

[8] R. L. Berger, E. A. Williams, A. Simon, Phys. Fluids B 1, 414 (1989)

[9] V. P. Silin, Zh. Eksp. Teor. Fiz. 47, 2254 (1994) [Sov. Phys. JETP 20, 1510 (1965); G. J. Pert, Phys. Rev. E 51, 4778 (1995)

[10] M. N. Rosenbluth, Phys. Rev. Lett., 29, 565 (1972)

[11] E. A. Williams, PRL 59, 2709 (1987); R. P. Drake and E. A. Williams, PRL 67, 2477 (1991)

[12] D. R. Nicholson and A. N. Kaufman, Phys. Rev. Lett. 33, 1207 (1974)

[13] D. S. Clark, PhD Thesis (Princeton University, 2003)

[14] G. J. Morales and T. M. O’Neil, Phys. Rev. Lett. 28, 417 (1972)

[15] A detailed examination of kinetic nonlinearities is beyond the scope of this paper but deserves further attention.

[16] The strong field reduction is not essential as it has an effect only in the initial stage of the heating. 


\section{External Distribution}

Plasma Research Laboratory, Australian National University, Australia

Professor I.R. Jones, Flinders University, Australia

Professor João Canalle, Instituto de Fisica DEQ/IF - UERJ, Brazil

Mr. Gerson O. Ludwig, Instituto Nacional de Pesquisas, Brazil

Dr. P.H. Sakanaka, Instituto Fisica, Brazil

The Librarian, Culham Laboratory, England

Mrs. S.A. Hutchinson, JET Library, England

Professor M.N. Bussac, Ecole Polytechnique, France

Librarian, Max-Planck-Institut für Plasmaphysik, Germany

Jolan Moldvai, Reports Library, Hungarian Academy of Sciences, Central Research Institute for Physics, Hungary

Dr. P. Kaw, Institute for Plasma Research, India

Ms. P.J. Pathak, Librarian, Institute for Plasma Research, India

Ms. Clelia De Palo, Associazione EURATOM-ENEA, Italy

Dr. G. Grosso, Instituto di Fisica del Plasma, Italy

Librarian, Naka Fusion Research Establishment, JAERI, Japan

Library, Laboratory for Complex Energy Processes, Institute for Advanced Study, Kyoto University, Japan

Research Information Center, National Institute for Fusion Science, Japan

Dr. O. Mitarai, Kyushu Tokai University, Japan

Dr. Jiangang Li, Institute of Plasma Physics, Chinese Academy of Sciences, People's Republic of China

Professor Yuping Huo, School of Physical Science and Technology, People's Republic of China

Library, Academia Sinica, Institute of Plasma Physics, People's Republic of China

Librarian, Institute of Physics, Chinese Academy of Sciences, People's Republic of China

Dr. S. Mirnov, TRINITI, Troitsk, Russian Federation, Russia

Dr. V.S. Strelkov, Kurchatov Institute, Russian Federation, Russia

Professor Peter Lukac, Katedra Fyziky Plazmy MFF UK, Mlynska dolina F-2, Komenskeho Univerzita, SK-842 15 Bratislava, Slovakia

Dr. G.S. Lee, Korea Basic Science Institute, South Korea

Institute for Plasma Research, University of Maryland, USA

Librarian, Fusion Energy Division, Oak Ridge National Laboratory, USA

Librarian, Institute of Fusion Studies, University of Texas, USA

Librarian, Magnetic Fusion Program, Lawrence Livermore National Laboratory, USA

Library, General Atomics, USA

Plasma Physics Group, Fusion Energy Research Program, University of California at San Diego, USA

Plasma Physics Library, Columbia University, USA

Alkesh Punjabi, Center for Fusion Research and Training, Hampton University, USA

Dr. W.M. Stacey, Fusion Research Center, Georgia Institute of Technology, USA

Dr. John Willis, U.S. Department of Energy, Office of Fusion Energy Sciences, USA

Mr. Paul H. Wright, Indianapolis, Indiana, USA 
The Princeton Plasma Physics Laboratory is operated by Princeton University under contract with the U.S. Department of Energy.

\author{
Information Services \\ Princeton Plasma Physics Laboratory \\ P.O. Box 451 \\ Princeton, NJ 08543
}

Phone: 609-243-2750

Fax: 609-243-2751

e-mail: pppl_info@pppl.gov

Internet Address: http://www.pppl.gov 